Annales Geophysicae (2003) 21: 869-879 (C) European Geosciences Union 2003

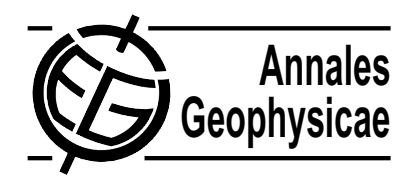

\title{
Substorm-associated large-scale magnetic field changes in the magnetotail: a prerequisite for "magnetotail deflation" events
}

\author{
H. Nakai ${ }^{1}$ and Y. Kamide ${ }^{2}$ \\ ${ }^{1}$ Ibaraki High School, Ibaraki, Osaka, Japan \\ ${ }^{2}$ Solar-Terrestrial Environment Laboratory, Nagoya University, Toyokawa, Japan
}

Received: 18 April 2002 - Revised: 22 July 2002 - Accepted: 20 August 2002

\begin{abstract}
An attempt is made to search for a critical condition in the lobe magnetic field to initiate large-scale magnetic field changes associated with substorm expansions. Using data from ISEE-1 for 1978, sudden decreases in the lobe magnetic field accompanied by magnetic field dipolarizations are identified. In this study, such events are designated as the magnetotail deflation. The magnetic field component parallel to the equatorial plane, $B_{E}$, is normalized to a fixed geocentric distance, $B_{E N}$, and is corrected for the compression effect of the solar wind dynamic pressure, $B_{E N C}$. It is shown that the $B_{E N C}$ value just prior to a magnetotail deflation correlates well with the Dst index; $B_{E N C}=37.5-0.217 D_{s t_{0}}$, where $D s t_{0}$ denotes the Dst value corrected for the solar wind dynamic pressure. This regression function appears to delineate the upper limit of $B_{E N C}$ values, when they are sorted by the $D s t_{0}$ index. On the basis of this finding it is suggested that a prerequisite condition for magnetotail deflations must exist in the magnetosphere.
\end{abstract}

Key words. Magnetospheric physics (magnetotail; current systems; storms and substorms)

\section{Introduction}

When the interplanetary magnetic field (IMF) is directed southward, Earth's magnetic flux is transported from the dayside magnetosphere to the magnetotail. This process enlarges the magnetotail diameter and increases the lobe magnetic field flux (Maezawa, 1975; Fairfield et al., 1981). Storing magnetic energy in the magnetotail, the magnetic configuration evolves toward an unstable state. Accumulated energy is eventually unloaded, resulting in a sudden decrease in the tail radius and magnetic field magnitude (Maezawa, 1975; Fairfield and Ness, 1970). This loading/unloading concept is corroborated by ground observations that the size of the polar cap increases prior to the expansion onset of substorms and decreases after the onset (e.g. Frank and Craven, 1988). The unloading process is successfully interpreted by the near-

Correspondence to: H. Nakai (nakai@ibaraki.osaka-c.ed.jp)
Earth neutral line (NENL) model, which also predicts the tailward ejection of plasmoids (e.g. Russell and McPherron, 1973; Hones et al., 1973). Ample evidence for the existence of plasmoids has been compiled to support the NENL model (e.g. Hones, 1979; Slavin et al., 1984; Ieda et al., 1998). Recently, measurements by Geotail revealed that the NENL is formed preferably in the region $X=-20--30 R_{E}$ (Nagai et al., 1998, and references therein).

Extensive efforts have been made to search for triggering actions for the unloading process (e.g. Burch, 1979). Northward turnings of the IMF have often been referred to as a trigger of substorm expansion onsets (e.g. Caan et al., 1975). Lyons et al. (1997) contended that the coincidence between these two phenomena is not by chance but has real significance. However, it does not mean that a triggering action always exists in the solar wind. McPherron et al. (1986) suggested that the expansion onset is caused by some inner process, which is influenced by changes in the solar wind. Given an internal particular condition for triggering the unloading process, it is natural to relate it to the energy and/or the magnetic flux stored in the magnetotail prior to the expansion onset. Baker et al. (1990) and Klimas et al. (1992) have attempted to understand the loading/unloading process on the basis of a dripping faucet analogy, assuming the existence of a threshold in the lobe magnetic field flux for the unloading process to occur. However, the critical value of the threshold in the real magnetotail was not identified in these works.

In this paper, we examine lobe magnetic field reductions that suddenly occur in association with the expansion onset of substorms. For convenience, we designate such magnetic field variations as the "magnetotail deflation", or simply the deflation. One of the difficulties in finding the critical condition for deflation events is attributed to the fact that they seem to occur regardless of the lobe magnetic field intensity, as shown in the later section. During the last decade the statistical behavior of the lobe magnetic field has been studied (Nakai et al., 1991, 1999; Nakai and Kamide, 1994; Fairfield and Jones, 1996; Ostapenko and Maltsev, 2000). They showed that the magnitude of the lobe magnetic field depends primarily on the solar wind pressure and geomagnetic activity represented by the $D s t$ index. On the basis 

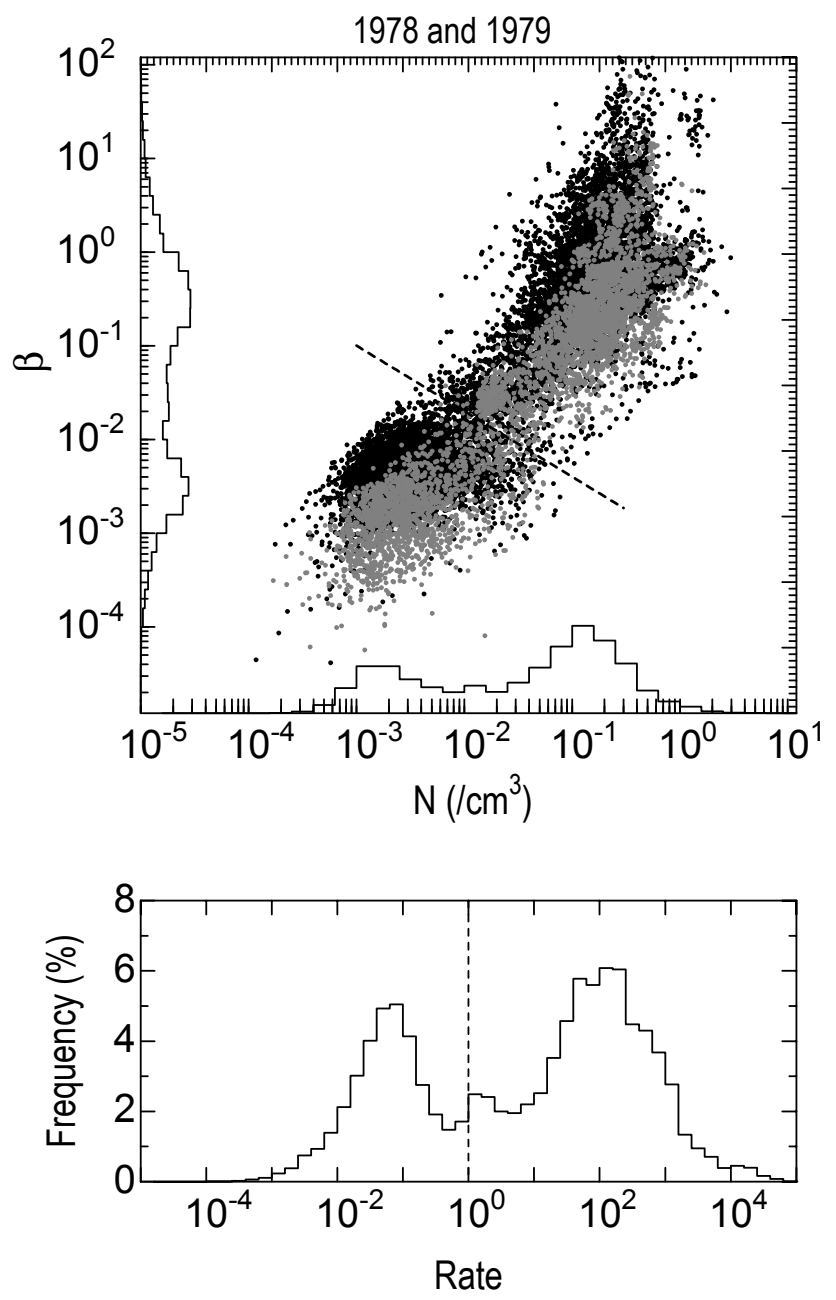

Fig. 1. Scatter plot of the plasma $\beta$ value as a function of the ion density $N$ in the region $|Y|<8 R_{E}$. Black and gray dots indicate the data points from the regions $S<15 R_{E}$ and $S \geq 15 R_{E}$, respectively, where $S$ denotes $\left(X^{2}+Y^{2}\right)^{1 / 2}$. The histograms for the $\beta$ value and the density are also shown along the vertical and horizontal axes, respectively. The gray line shows the equation, $\beta=8.0 \times 10^{-4} N^{-0.70}$. Data points located below this line are regarded as those obtained in the lobe. The histogram of the ratio of $\beta$ to the right-hand side of this equation is shown in the bottom panel.

of these works, the critical condition for the deflation event is searched for in this study. A clear deflation event is first examined in detail. A statistical study is then performed to show that the deflation event tends to occur when the lobe magnetic field reaches a critical value that is a function of the solar wind pressure and the $D s t$ index.

\section{Data and procedure}

The ISEE-1 spacecraft was launched on 22 October 1977, into an orbit with apogee at about $23 R_{E}$ geocentric distance, perigee at about $300 \mathrm{~km}$ altitude, an inclination of 29 degrees, and an orbital period of $57 \mathrm{~h}$. Magnetic field and ion density

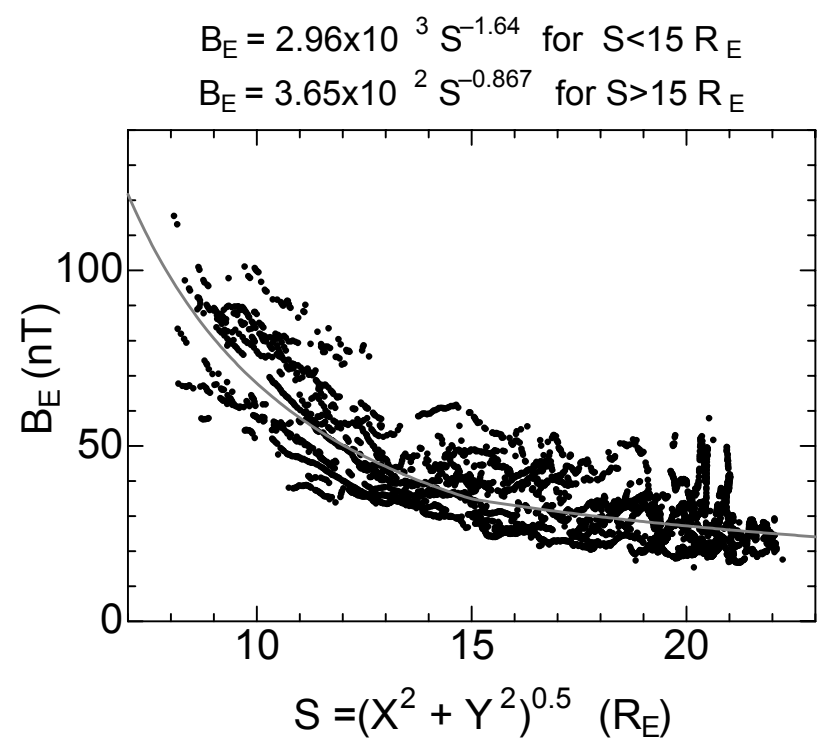

Fig. 2. The lobe magnetic field component $B_{E}$ plotted as a function of $S . B_{E}$ and $S$ are defined as $\left(B_{x}^{2}+B_{y}^{2}\right)^{1 / 2}$ and $\left(X^{2}+Y^{2}\right)^{1 / 2}$, respectively. The gray lines show the regression lines obtained separately for $S<15 R_{E}$ and $S \geq 15 R_{E}$.

data for 1978-1979 are used in the present study: see Russell (1978) and Shelley et al. (1978), respectively, for technical details of these experiments. Solar wind and IMF parameters obtained by IMP 8 are also used, taking into account time lags from IMP 8 to Earth.

The top panel in Fig. 1 shows the $\beta$ value plotted as a function of the ion density $N$ in the midnight region $|Y|<8 R_{E}$. Black and gray dots indicate the data points from the regions $S<15 R_{E}$ and $S \geq 15 R_{E}$, respectively, where $S$ denotes $\left(X^{2}+Y^{2}\right)^{1 / 2}$. The histograms for the $\beta$ value and the density are also shown along the vertical and horizontal axes, respectively. Two peaks are seen in both histograms. The "low$\beta$ and low-density" peak and the "high- $\beta$ and high-density" peak correspond to the tail lobe and the central plasma sheet, respectively. However, the separation between these two categories is not very clear. This ambiguity probably results from the dependence of these quantities on the distance $S$, indicated by the displacement between black and gray data points. Instead, it is seen that the scatter plots are separated more clearly by a dashed line, which is represented by

$\beta=8.0 \times 10^{-4} N^{-0.70}$.

The histogram of the ratio of $\beta$ to the right-hand side of this equation is shown in the bottom panel of Fig. 1. Data points can evidently be separated into two characteristic categories. In the bottom panel, there is a small peak adjacent to the unity. This peak probably corresponds to the plasma sheet boundary layer. Consequently, data points below the line of the Eq. (1) in the top panel are regarded as those from the tail lobe.

The lobe magnetic field depends on the distance from Earth, the solar wind pressure, and magnetic storm activity 




Fig. 3. Dependence of the solar wind dynamic pressure $P_{D}$ on the $B_{E N}$ value: see the text for the definition of $B_{E N}$. Data points are divided into three groups according to the $D s t_{0}$ index; (a) for $D s t_{0} \geq-30 \mathrm{nT}$, (b) for $-60 \leq D s t_{0}<-30 \mathrm{nT}$, and (c) for $D s t_{0}<$ $-60 \mathrm{nT}$. The gray dots in the panel (c) are for $D s t_{0}<-90 \mathrm{nT}$. The solid lines show the regression function of $P_{D}^{0.5}$ for $B_{E N}$.

represented by Dst (e.g. Nakai et al., 1999). Thus, we must eliminate the effect of these quantities from the lobe field intensity to search for an internal condition for triggering unloading processes. The magnetic field component $B_{E}$ is plotted as a function of $S$ in Fig. 2, where $B_{E}=\left(B_{x}^{2}+B_{y}^{2}\right)^{1 / 2}$ in solar magnetospheric coordinates. Since the $B_{E}$ component closely reflects the intensity of the neutral sheet current near the spacecraft, $B_{E}$ is used through the present study, instead of the total magnitude of the lobe field. When the lobe field magnitude is fitted by a power function of the radial distance from Earth, the power of the radial distance is found to be smaller in the absolute value for regions farther from Earth. Thus, in this study, data points are divided into two groups at $S=15 R_{E}$. As a result, they are approximated by

$B_{E}=2.96 \times 10^{3} S^{-1.64} \quad$ for $S<15 R_{E}$,

and

$B_{E}=3.65 \times 10^{2} S^{-0.867} \quad$ for $S \geq 15 R_{E}$,

as shown by solid lines in Fig. 2. Then, the $B_{E}$ value normalized at $S=15 R_{E}$ is defined as $B_{E N}=B_{E}(S / 15)^{\delta}$ with
21 March 1978

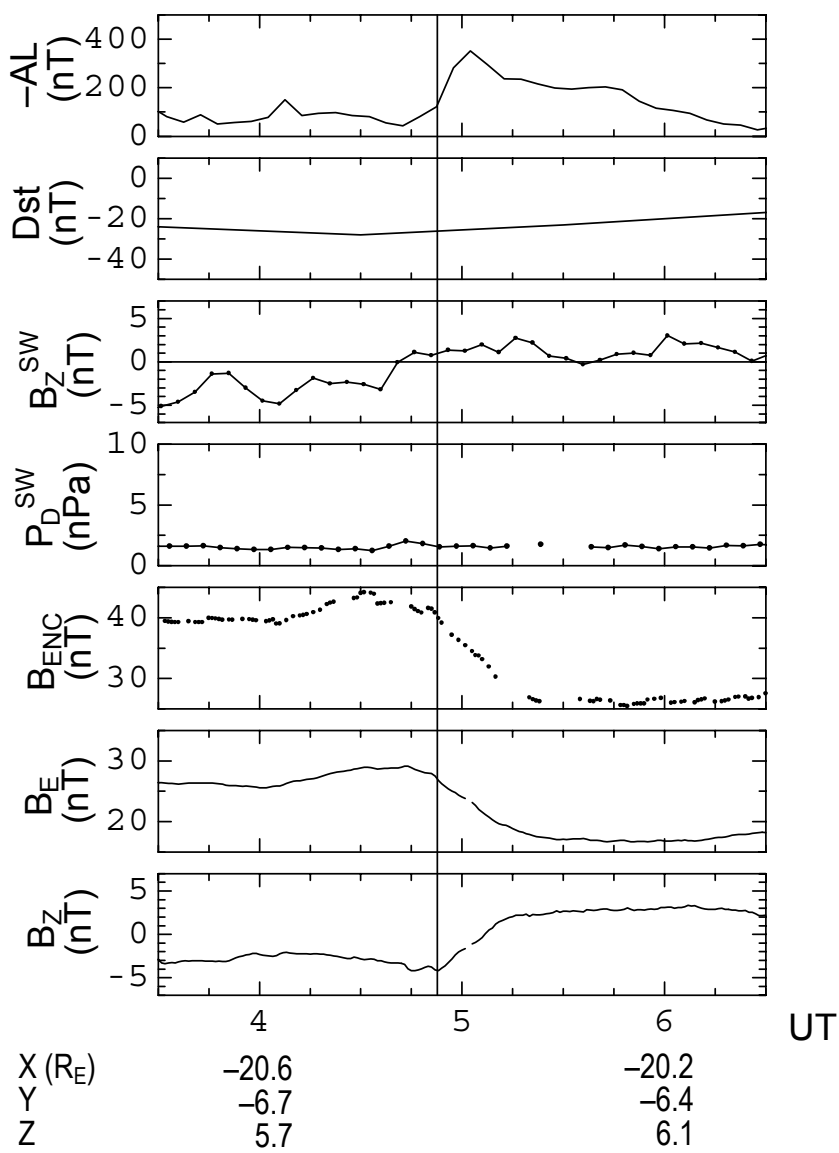

Fig. 4. An example of magnetotail deflation (deflation) events. From top to bottom, the $A L$ and $D s t$ indices, the IMF $B_{z}$ component, the solar wind dynamic pressure, and the magnetic field components $B_{E N C}, B_{E}$, and $B_{z}$ observed on 21 March 1978 are plotted.

$\delta=1.64$ and 0.867 for $S<15 R_{E}$ and $S \geq 15 R_{E}$, respectively.

How $B_{E N}$ relates to the solar wind dynamic pressure $P_{D}$ is shown in Figs. 3a-c. Data points are divided into three groups, according to the $D s t_{0}$ index, which is the pressurecorrected Dst value (Burton et al., 1975; Valdivia et al., 1996), defined as

$D s t_{0}(\mathrm{nT})=D s t-10.5 P_{D}^{0.5}+22$.

The solid lines show the regression function of $P_{D}^{0.5}$ for $B_{E N}$. The regression slopes are 13.0, 10.6, and 9.3 for Dst $t_{0} \geq-30 \mathrm{nT},-60 \leq$ Dst $_{0}<-30 \mathrm{nT}$, and Dst $t_{0}<$ $-60 \mathrm{nT}$, respectively. The slope is less steep for larger $\left|D s t_{0}\right|$. To confirm this tendency further, $B_{E N}$ values with $D s t_{0}<-90 \mathrm{nT}$ are plotted in gray dots in Fig. 3c. In this case the plots show that there is no correlation between these two quantities. Since the changes in the regression slope are relatively small for $D s t \geq-90 \mathrm{nT}$, the normalization of $B_{E N}$ 


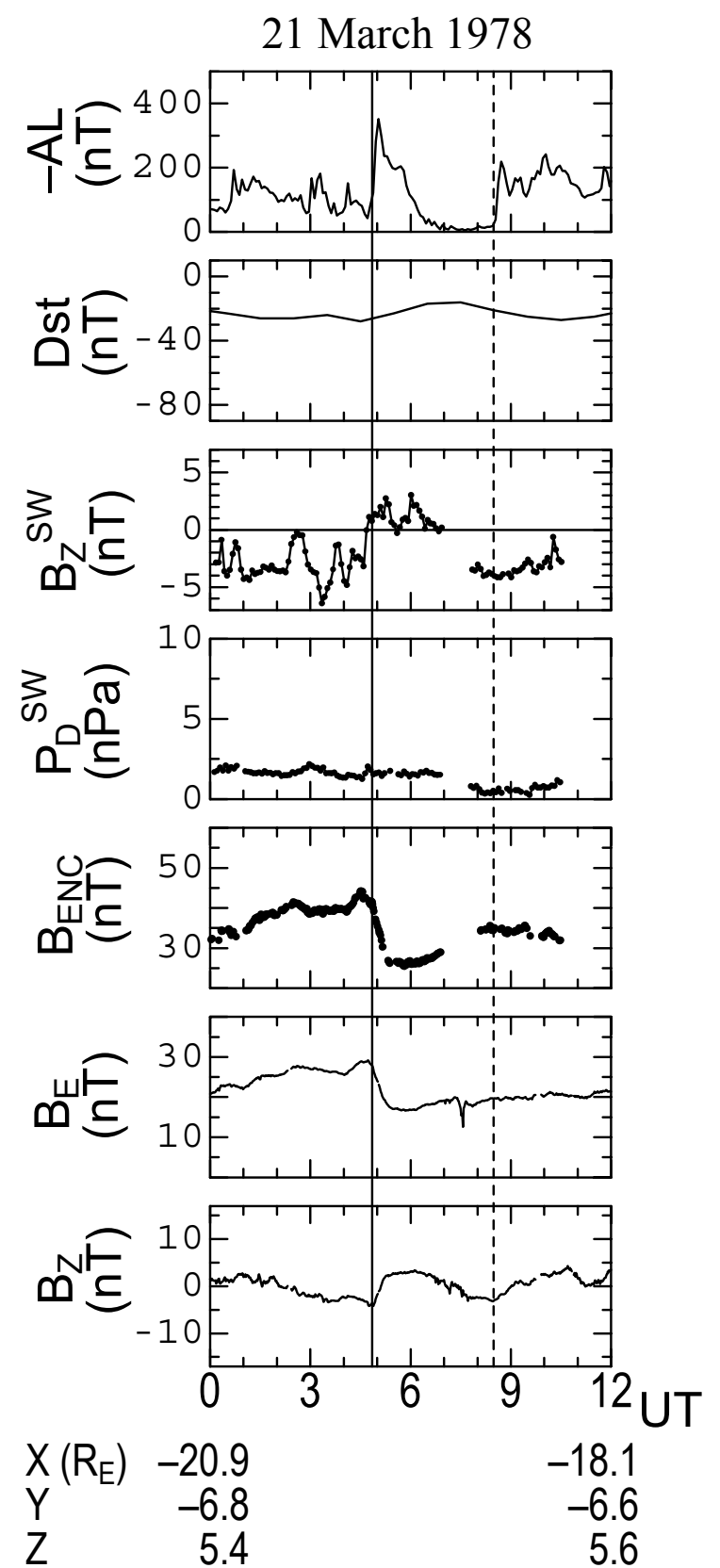

Fig. 5. Deflation events sampled in the present study in the same format as Fig. 4. The onset times of the magnetic field dipolarizations associated with the deflations are indicated by solid lines. The dashed lines show the magnetic field dipolarizations without the deflation.

values with respect to the solar wind pressure is made for the $-90 \leq D s t_{0}<30 \mathrm{nT}$ range in the following way:

$B_{E N C}=B_{E N}-10.0\left(P_{D}^{0.5}-\left\langle P_{D}\right\rangle^{0.5}\right)$

where the average of the solar wind dynamic pressure, $\left\langle P_{D}\right\rangle$, for $1978-1979$ is taken to be $2.50 \mathrm{nPa}$ (Nakai and Kamide, 1994).

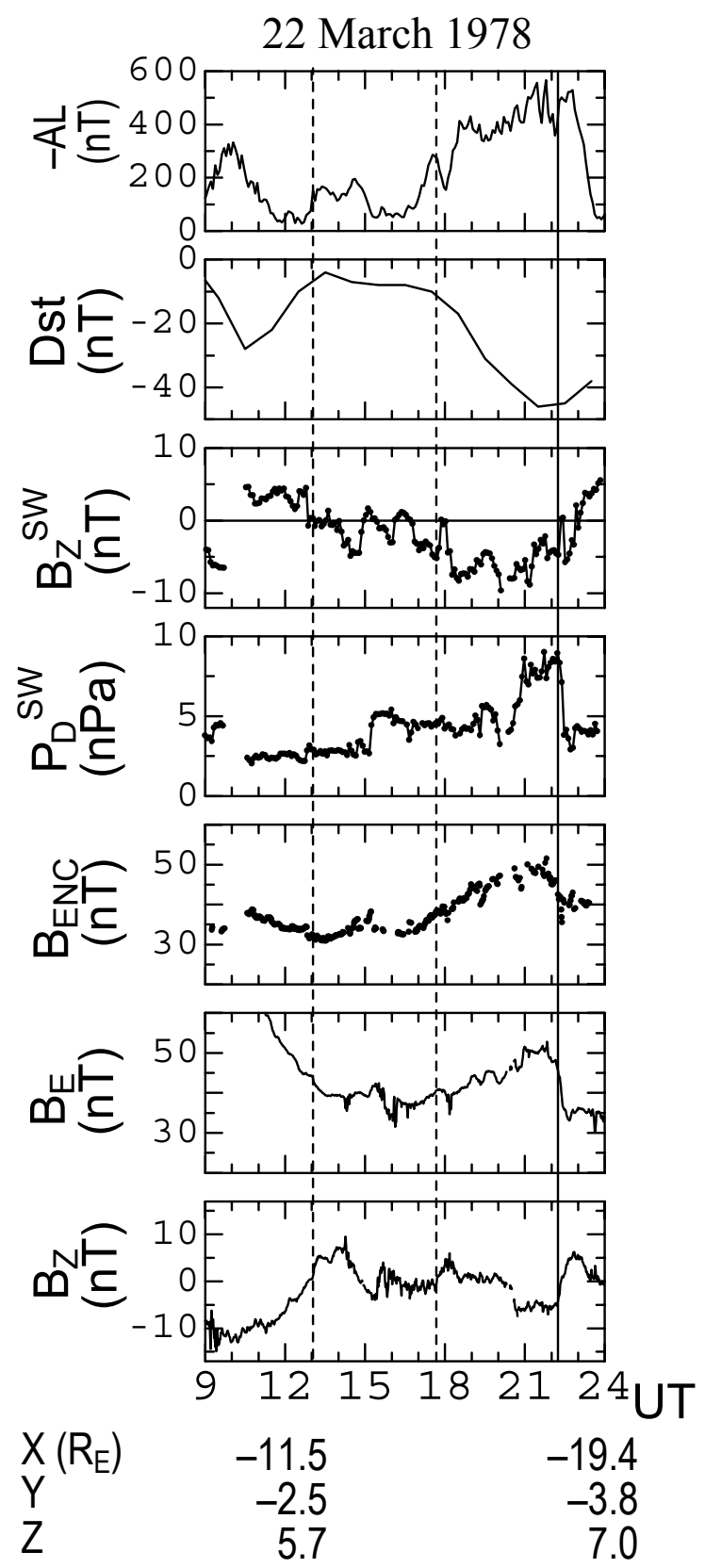

Fig. 5. Continued .....

\section{Results}

We have searched for intervals during each of which more than ninety 1-min data points of $B_{E N C}$ were available. As a result, thirteen days of data have been sampled from 1978. Unfortunately, none of the data from 1979 have met this condition, primarily because ISEE-1 passed through the tail lobe less frequently in 1979 than in 1978. Inspecting these thirteen days by sight, fourteen clear deflation events have been identified. In all of these events the $B_{E N}$ value shows a sudden decrease by more than $5 \mathrm{nT}$ within $40 \mathrm{~min}$ from the onset, and is accompanied by a magnetic field dipolarization.

One typical example that occurred on 21 March 1978 is 
April 3-4, 1978

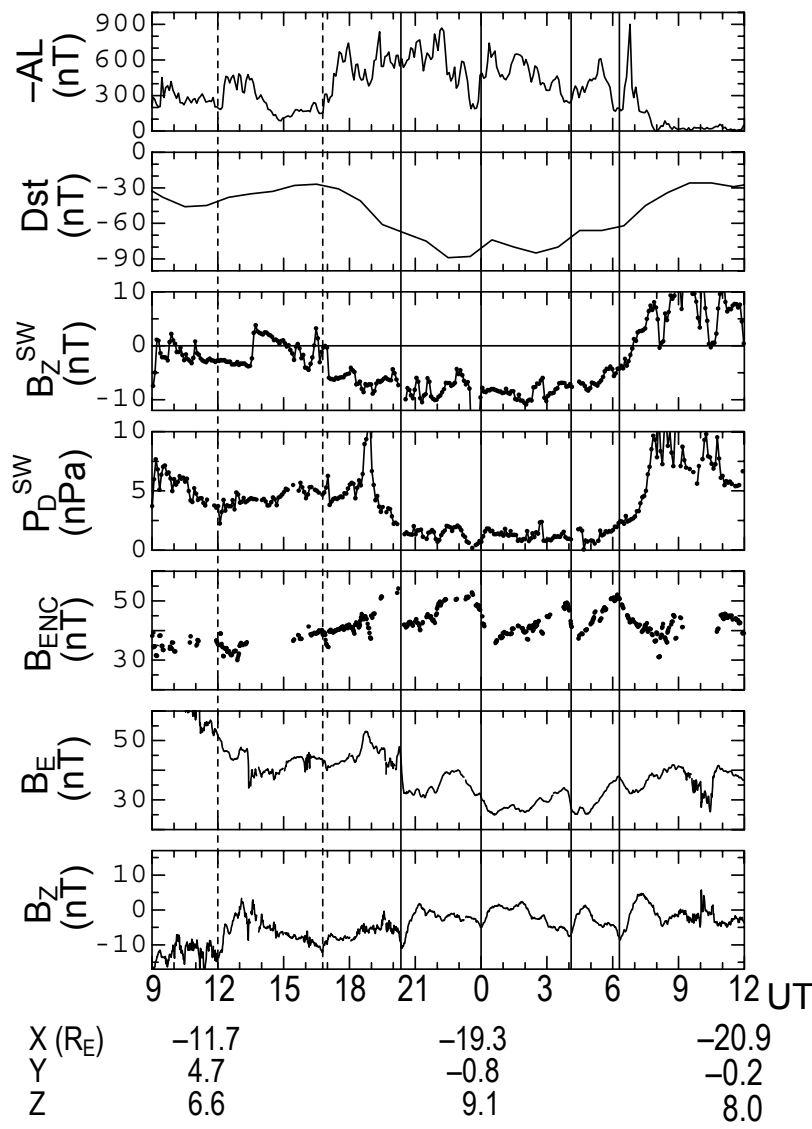

Fig. 5. Continued .....

displayed in Fig. 4, where the $B_{E N C}$ and $B_{z}$ values, the geomagnetic indices, $A L$ and $D s t$, and the solar wind parameters, $B_{z}^{s w}$ and $P_{D}^{s w}$, are plotted. The ISEE-1 position in solar magnetospheric coordinates is shown at the bottom. The time when $B_{z}$ began to increase is marked by a solid line. The 5min IMF data are shifted in time considering the traveling time of the solar wind from IMP 8 to Earth, assuming that the solar wind speed is $400 \mathrm{~km} / \mathrm{s}$, the angle between the SunEarth line and the magnetic field line is 45 degrees. Since IMP 8 was located around $(21,11,-13) R_{E}$, and the IMF was actually pointing between 03:00 LT and 06:00 LT during the period concerned, the traveling time of the IMF can be 2-3 min shorter than those adopted in Fig. 4. According to the definition, $B_{E N C}$ values can be plotted only for intervals when ISEE-1 was in the lobe region and IMP 8 data were available. Geomagnetic activity was rather low in terms of the $D s t$ index during this particular interval. A clear dipolarization occurred at 04:53 UT, accompanied by an enhancement in the $A L$ index. The $B_{E N C}$ component was in the tendency of increasing until $\sim 04: 30$ UT due to the prolonged southward IMF (see Fig. 5). The $B_{z}$ component of the IMF turned northward at 04:40 UT. The $B_{E N C}$ value began to decrease at 04:51 UT, and continued decreasing until 05:23 UT. The amount of the decrease reached $15.3 \mathrm{nT}$, resulting in a

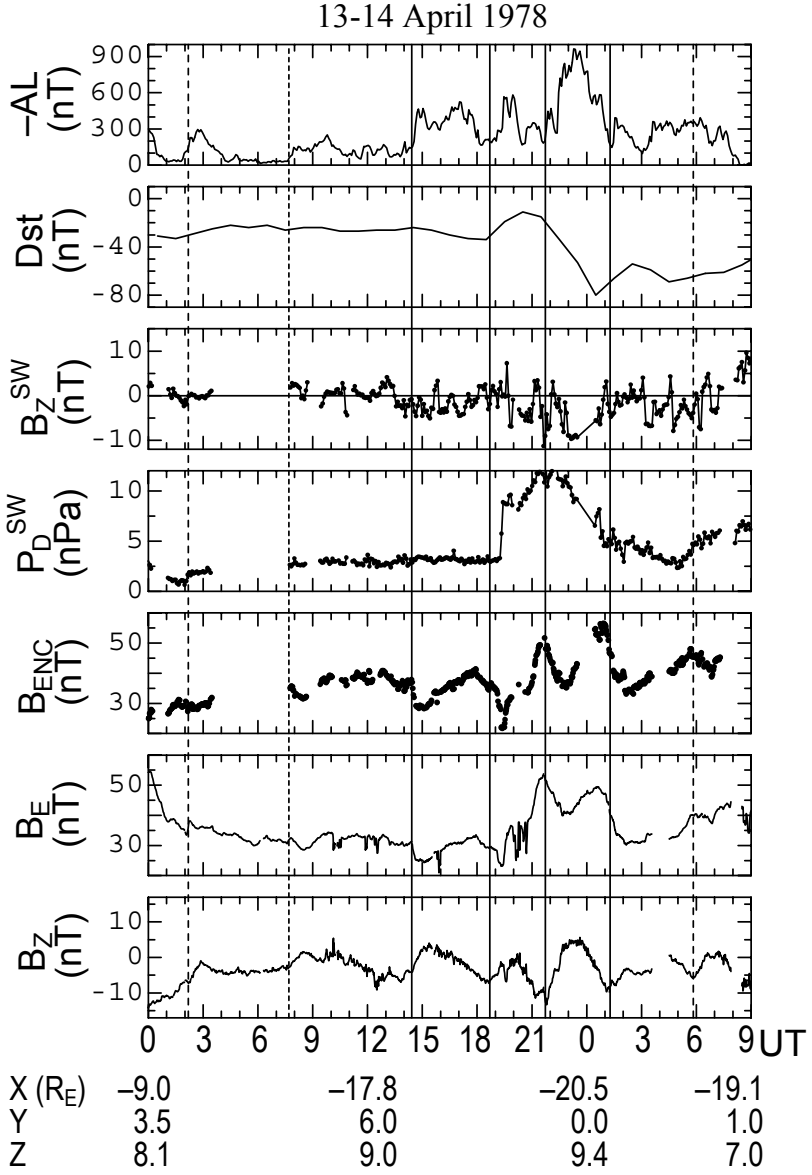

Fig. 5. Continued .....

$60 \%$ reduction in the total pressure. The two-minute delay of the dipolarization onset from the decrease in $B_{E N C}$ indicates that a reduction in the neutral sheet current occurred beneath the spacecraft and subsequently extended tailward.

Entire events sampled in this study are shown in Fig. 5 in the same format as in Fig. 4. The vertical solid lines indicate the deflation events. All the deflation events, except one at 14:40 UT on 15 April, were observed in the region $X<-17 R_{E}$. Histograms of $B_{E N}$ values in the whole data set and those at the beginning of deflation events are shown in Fig. 6. It is noticed that the deflation events are distributed in the wide range of $B_{E N}$ values between $30 \mathrm{nT}$ and $70 \mathrm{nT}$, although they tend to occur with relatively large $B_{E N}$ values. As mentioned in the earlier section, the question why the lobe magnetic field can take such a wide range in its magnitude at deflation events is the particular motivation of the present study.

It is noticed in Fig. 5 that there are magnetic field dipolarizations without any evident decreases in $B_{E N C}$. Such fifteen events are marked by dashed vertical lines in the figure. As in the deflation events, these dipolarizations seem to be generally associated with enhancements in the $A L$ index. These events will be discussed briefly in the later section.

In Fig. $7, B_{E N C}$ values at the beginning of the deflation 


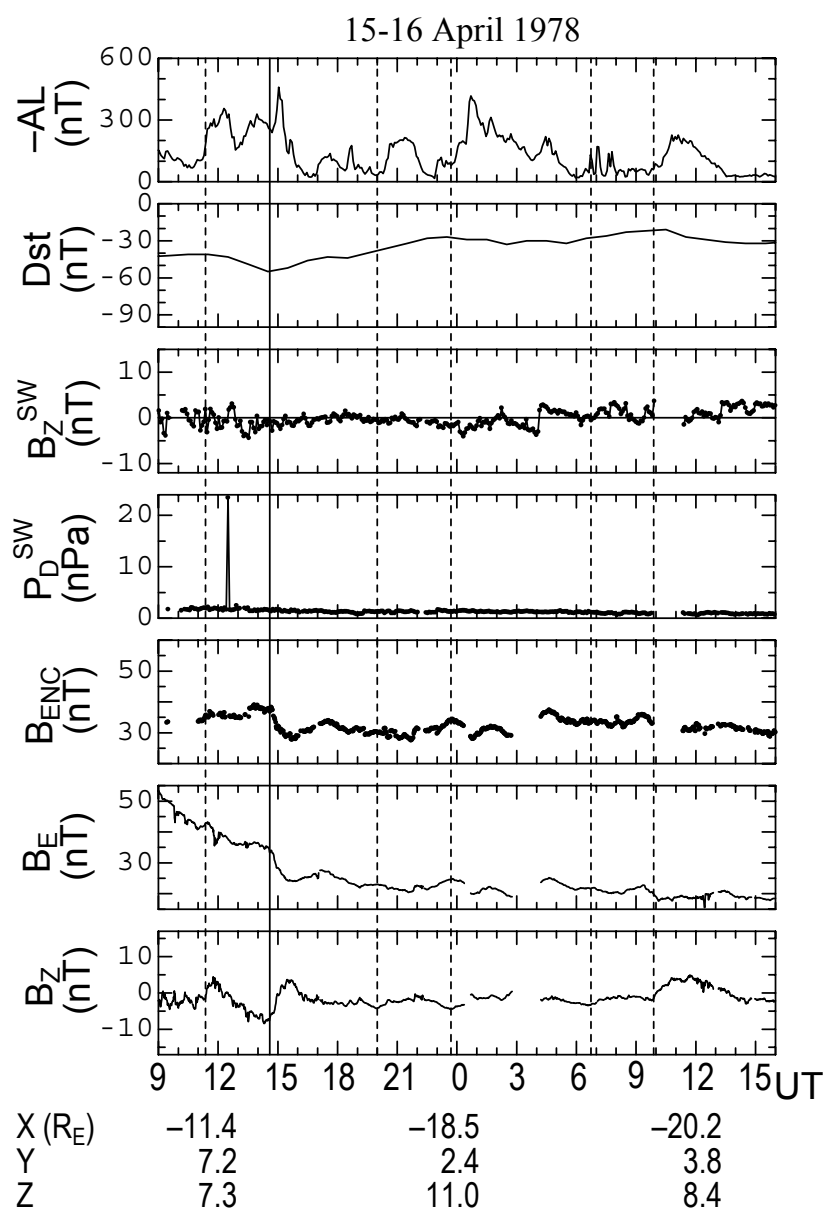

Fig. 5. Continued .....

events are plotted by solid circles as a function of $D s t_{0}$ along with entire $B_{E N C}$ values plotted by gray dots. The regression equation for gray dots is

$B_{E N C}=32.1-0.15$ Dst $_{0}$,

with the correlation coefficient of 0.56. The dashed line shows the regression line for the solid circles, which is given by

$B_{E N C}=37.5-0.217$ Dst $_{0}$.

The correlation coefficient is found to be 0.72 . This line approximately corresponds to the upper limit of $B_{E N C}$ for given $D s t_{0}$ values.

The solid circle at $D s t_{0}=-47 \mathrm{nT}$ and $B_{E N C}=38.3 \mathrm{nT}$ appears to be separated from the other set of solid circles in Fig. 7. This event was in fact observed at 14:40 UT on 15 April at $X=-13.5 R_{E}$, spatially separated from all other deflation events observed beyond $X=-17 R_{E}$. Variations in the IMF are plotted in Fig. 8 for the period around this event. A clear negative pulse in the magnetic field magnitude with an interval of about $10 \mathrm{~min}$ is seen prior to the onset of this event. The Earth's open magnetic field flux might be reduced during and/or after the pulse. Although it is difficult to

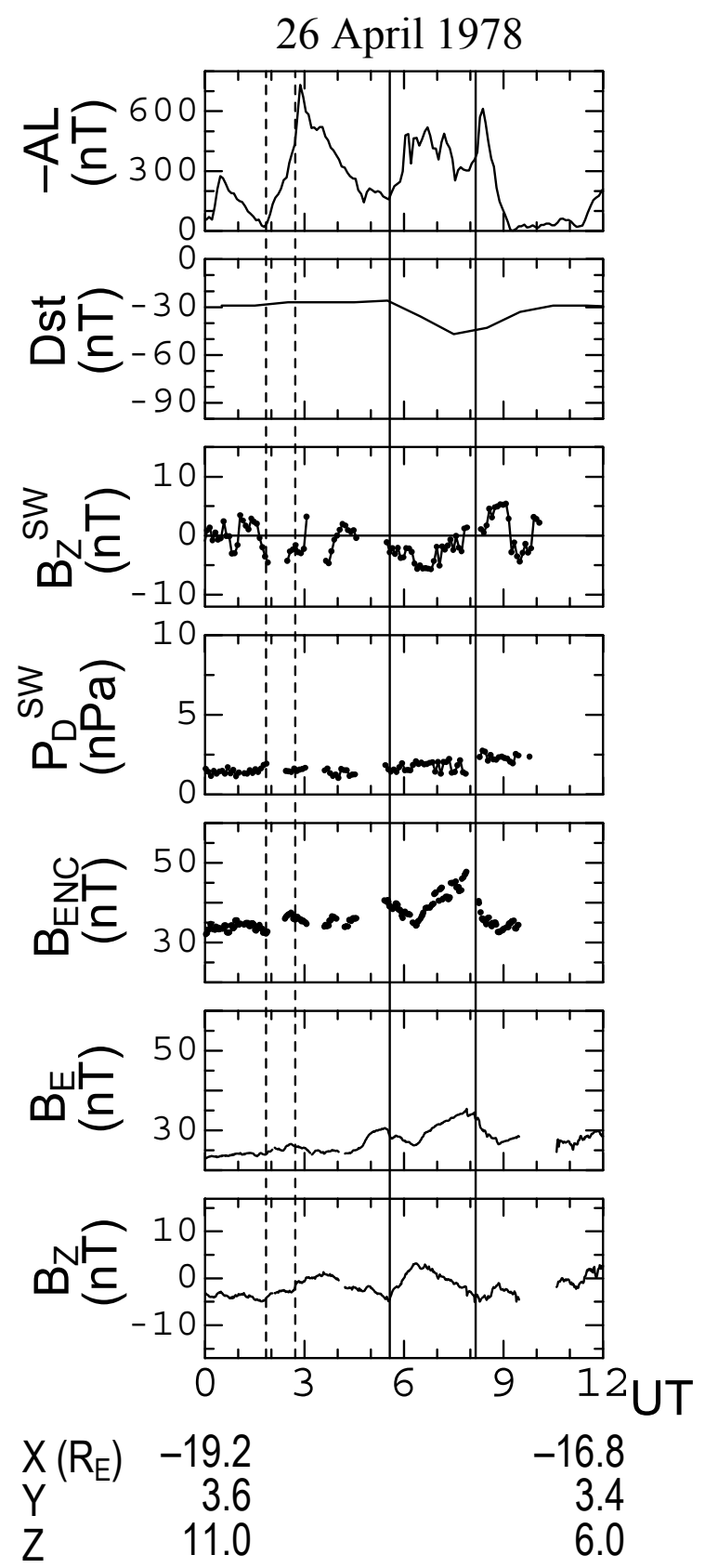

Fig. 5. Continued .....

identify the cause of the magnetic field decrease at 14:40 UT, this event can be regarded as an exceptional case.

Assuming that the $B_{E N}$ value at the onset time of the deflation event can be represented by a linear function of $P_{D}$ and Dst, the most appropriate coefficients have been obtained as

$B_{E N}=8.0-0.26 D s t+17 P_{D}^{0.5}$,

where the correlation coefficient is 0.95 . To see average behavior of the deflation events, the $B_{E}$ values in the fourteen deflation events in Fig. 5 are normalized at $S=20 R_{E}$, Dst $=-50 \mathrm{nT}$ and $P_{D}=2.5 \mathrm{nPa}$, using Eqs. (3) and (8). Since deflation events are preferably observed near the 


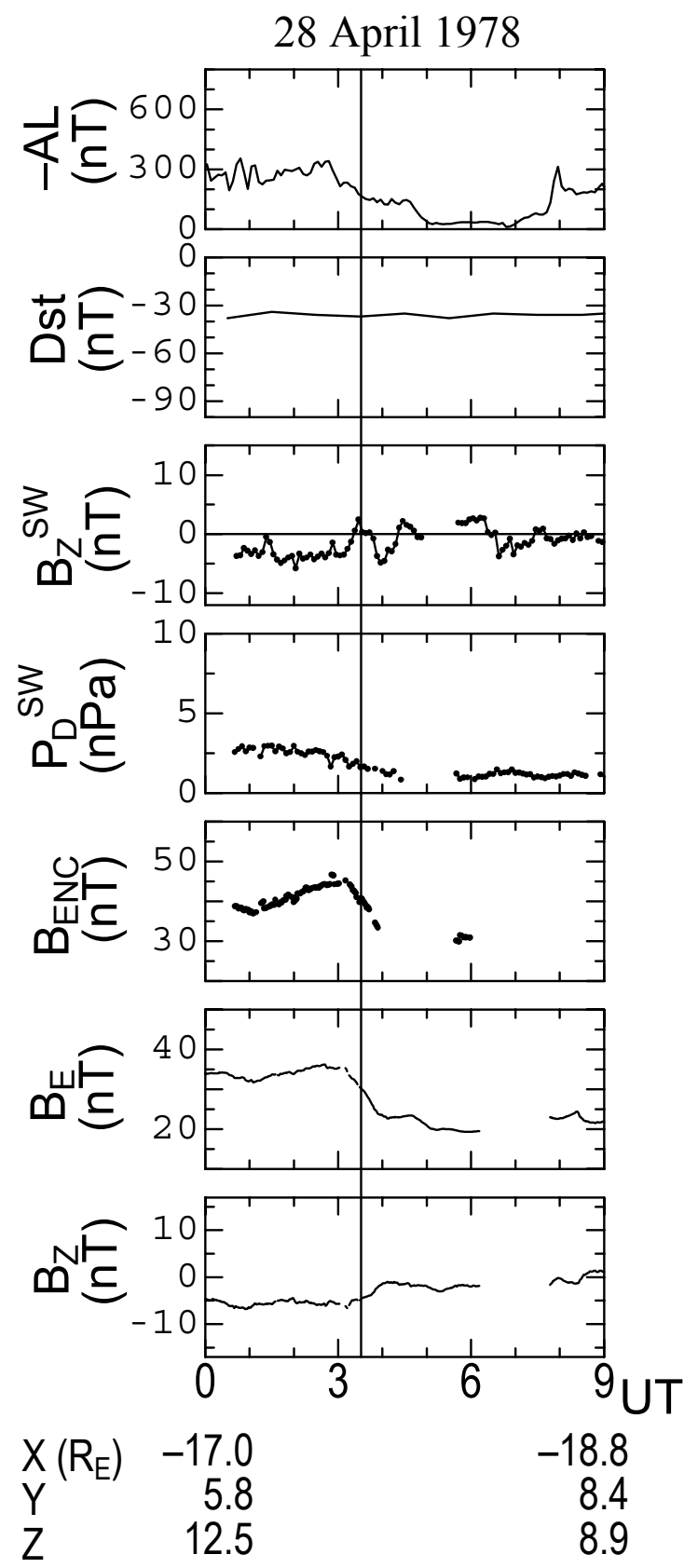

Fig. 5. Continued .....

apogee of the spacecraft in the present study, the distance for this normalization is chosen to be $S=20 R_{E}$. The upper panel of Fig. 9 shows the normalized $B_{E}$ values, superposing data with respect to the onset time of deflation events. The average $B_{E}$ just before the deflation is $36.8 \mathrm{nT}$, as shown by a larger solid circle. It is noticed that the $B_{E}$ component increases slowly by $0.04 \mathrm{nT} / \mathrm{min}$ (or $2.5 \mathrm{nT} / \mathrm{hr}$ ) for more than $2 \mathrm{~h}$ prior to the onset of deflation events, and it decreases by $0.3 \mathrm{nT} / \mathrm{min}$ for $\sim 30 \mathrm{~min}$ after the onset. The superposed change in the $B_{z}$ component is also shown at the bottom panel of Fig. 9. It is seen that the $B_{z}$ component decreases in association with the increase of the $B_{E}$ component. The

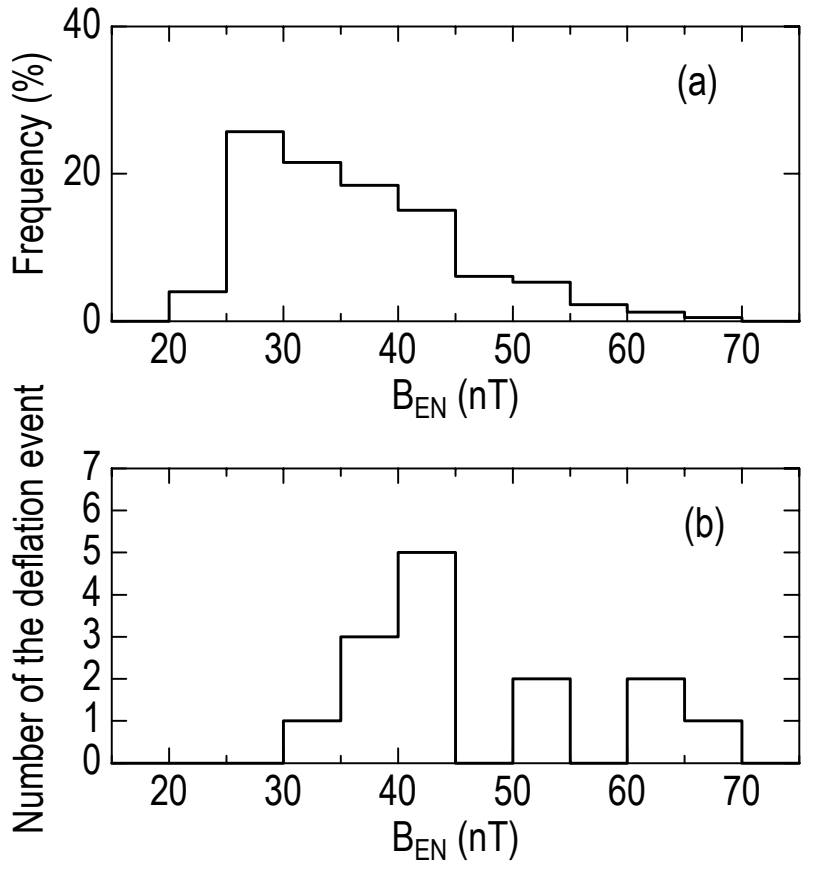

Fig. 6. (a) Occurrence frequencies of $B_{E N}$ values in percent. (b) Number of deflation events for each bin of the $B_{E N}$ value.

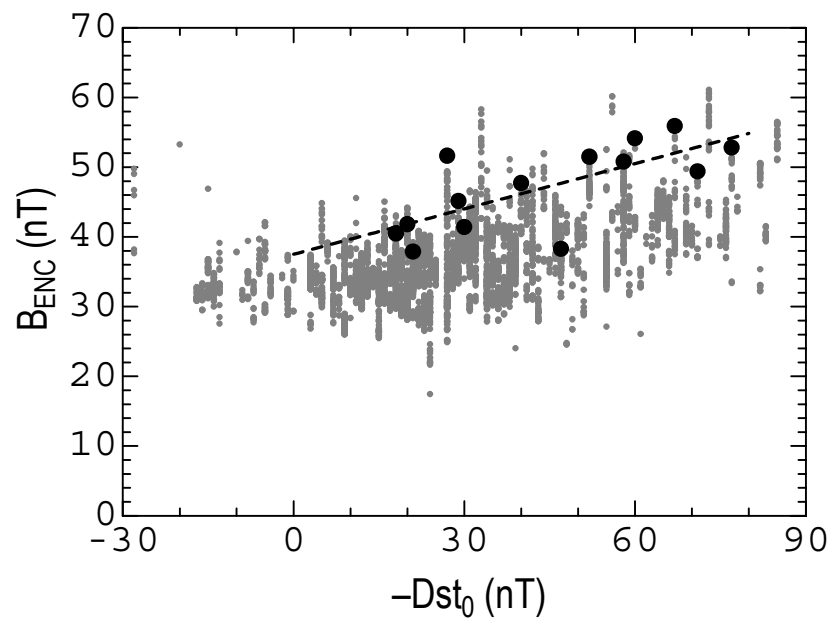

Fig. 7. The $B_{E N C}$ values at the onset time of the deflation event shown by solid circles as a function of $-D s t_{0}$. The gray dots are the whole data set for $-90 \leq D s t_{0}<30 \mathrm{nT}$. The dashed line shows the regression line for the solid circles, $B_{E N C}=37.5-0.217$ Dst $_{0}$. The correlation coefficient is 0.72 .

dipolarization, however, tends to be delayed by several minutes, as noted above for the 21 March event. The increment in the $B_{z}$ component continues for about $50 \mathrm{~min}$ after the dipolarization. 




Fig. 8. Variations in the IMF around the 14:40 UT event on 15 April 1978.

\section{Discussion}

Magnetotail deflation events were examined to search for a critical condition to initiate large-scale magnetic field changes in the lobe. It has been shown that the $B_{E N C}$ value just prior to deflation events correlates well with the Dst index. The regression function of $B_{E N C}$ appears to delineate the upper limit of data points in the $B_{E N C}-D s t_{0}$ scattering plot (see Fig. 7).

\subsection{A prerequisite condition for deflation events}

Seeing Fig. 7, some of the readers may argue that the lobe magnetic field usually reaches a peak before substormassociated decrease, known as the final stage of the so-called loading process. Because the authors use such a peak to identify the beginning of deflation events, it is natural that the deflation events should appear near the upper limit of the lobe intensity. $B_{E N C}$ has a correlation with $D s t$, and, therefore, the deflation events, which appear near the upper limit in the $B_{E N C}$ plot, must naturally show a correlation with $D s t$.
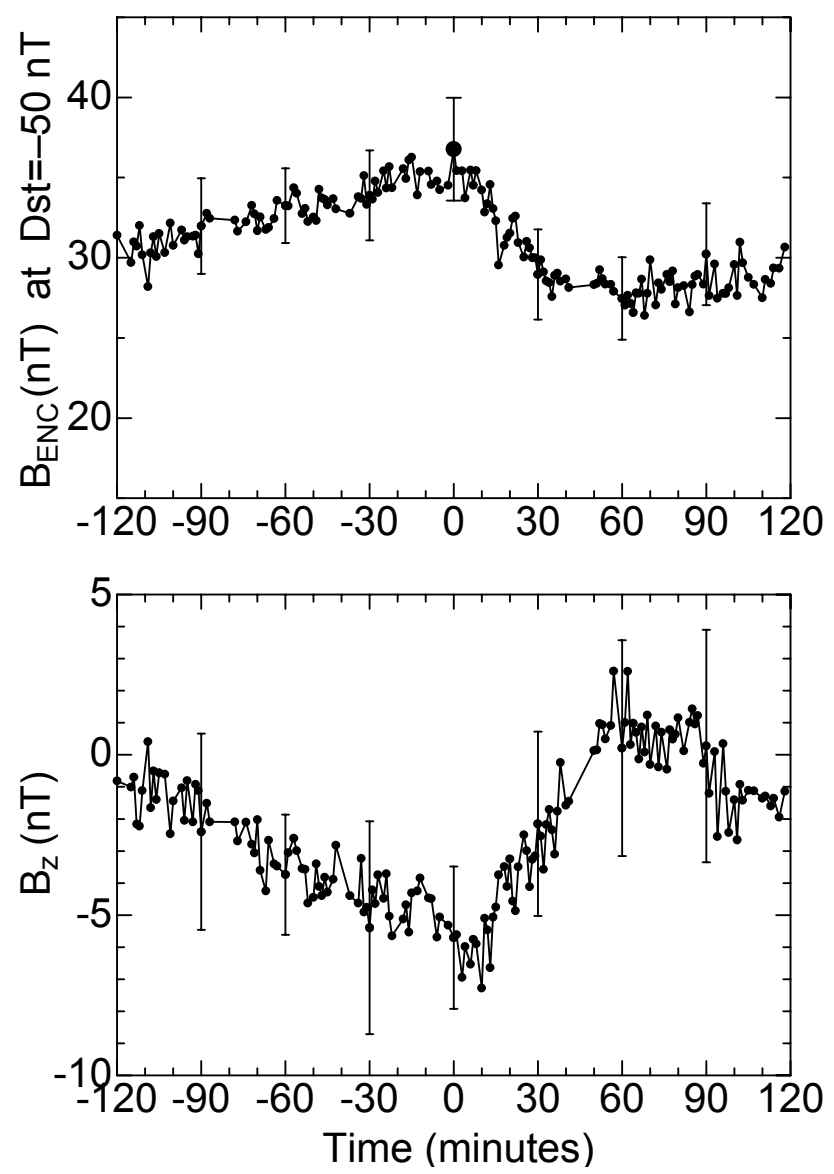

Fig. 9. Top: Superposed $B_{E}$ trace, constructed by averaging 14 deflation events with respect to the onset times of the deflation events. Using Eqs. (3) and (7), $B_{E}$ values are normalized to the values at $S=20 R_{E}$ and for $D s t=-50 \mathrm{nT}$ and $P_{D}=2.5 \mathrm{nPa}$. The average of normalized $B_{E}$ values, $36.8 \mathrm{nT}$, just prior to the onset times is shown by a larger solid circle.

Bottom: Superposed $B_{z}$ trace for the 14 deflation events. On both panels the vertical lines show the standard deviation every $30 \mathrm{~min}$.

As we show in Fig. 6, the $B_{E}$ values just prior to deflation events tend to appear in the upper half range of the whole data set. The method of the data sampling is actually responsible for this feature. However, the coincidence that is seen between the $B_{E N C}$ values just prior to the deflation event and the upper limit of data points in Fig. 7 is not explained in terms of the sampling methodology. Then, what relationship between the Dst and $B_{E N C}$ values is needed to realize this coincidence? It is important to note that the time scale of variations in the $D s t$ index is generally much longer than that of the lobe field intensity. For example, the traces of $B_{E N C}$ on 21 March and 3-4 April 1978, are plotted as a function of $D s t_{0}$ in Fig. 10. One and four deflation events occurred, respectively, during these periods. Assuming that the hourly $D_{s t} t_{0}$ value indicates the value at $30 \mathrm{~min}$ of every UTh, the $D s t_{0}$ value at each minute is derived by interpolating the hourly values. The thin and thick lines trace the variations of the $B_{E N C}$ value between 16:00 and 24:00 UT on 3 April and 
between 00:00 and 08:00 UT on 4 April, respectively. During the former (or latter) period, the magnetic storm was in the expansion (or recovery) phase. A gray line indicates the trace of $B_{E N C}$ during the 21 March event, which is shown in an extended scale in Fig. 4. The $B_{E N C}$ value changes dramatically before and after the deflation events. During these variations the $B_{E N C}$ value seems to be independent of the $D s t_{0}$ index. On the other hand, the traces approximately follow the regression Eq. (6) during the entire period, except the intervals around the deflation events. Therefore, it is suggested that there are two components in the variations of $B_{E N C}$, i.e. the $D s t$ dependent and Dst independent components.

We are able to estimate the $D s t$ dependent component on the basis of Tsyganenko's 89c (1989) magnetic field model (T89). The T89 model calculates separately the magnetic field contributions from the tail current system, the magnetopause closure current system, the ring current system, the Chapman-Ferraro current, and residual current systems for an arbitrary value of the $K_{p}$ index. The $X$ component of the magnetic field at $(-15,0,5) R_{E}$ is 23.7 and $38.6 \mathrm{nT}$ with $K_{p}=0 / 0+$ and $4-/ 4 / 4+$, respectively, while the contributions from the ring current system are 2.0 and $9.1 \mathrm{nT}$. Here, the time is set at 00:00 UT on 4 April 1978. Subtracting the dipole component, the averages of the $Z$ components at $(-1,0,0)$ and $(1,0,0) R_{E}$ on the Earth's surface are $-30.8\left(K_{p}=0 / 0+\right)$ and $-75.9 \mathrm{nT}\left(K_{p}=4-/ 4 / 4+\right)$. Assuming that the predicted field with $K_{p}=0 / 0+$ represents the quiet condition $(D s t=0)$, the nominal $D s t$ value for the assumed condition with $K_{p}=4-/ 4 / 4+$ is estimated to be about $-60 \mathrm{nT}$. Here, the magnetic field decrease at the Earth's surface is multiplied by 1.3, taking the effect of the Earth's interior induced currents into account (Langel et at., 1985). Therefore, the lobe magnetic field is estimated to increase by $\sim 15 \mathrm{nT}$ in association with a $60 \mathrm{nT}$ decrease in Dst. About $50 \%$ of this increase is attributed to an enhancement in the ring current. The residual comes from the tail current system and the magnetopause closure current system.

The finding that the Dst index is not influenced by the deflation event seems to contradict the aspect that the cross tail current can contribute $20-50 \%$ of the Dst index (e.g. Turner et al., 2000). From Fig. 9 it is inferred that the tail current decreases by about $30 \%$ on average at the deflation event. Given the $35 \%$ contribution from the tail current, the Dst index must increase $10 \%$ in association with the deflation event. A careful statistical analysis is required to detect this increment. In the above calculations with the T89 model, the contribution from the ring current system, the tail current system, the magnetopause closure current system and the residual current systems, mainly the Chapman-Ferraro current, are $-25.0,-28.2,2.1$, and $20.3 \mathrm{nT}$ at the Earth's surface with $K_{p}=0,0+$, and $-75.0,-44.5,15.9$, and $27.7 \mathrm{nT}$ with $K_{p}=4-/ 4 / 4$, respectively. These values imply that an increase in the contribution from the tail current can be partly offset by an increasing influence from the magnetopause closure current. To our knowledge, this effect has not been discussed in previous papers. If the magnetopause closure current decreases in association with the reduction of the tail

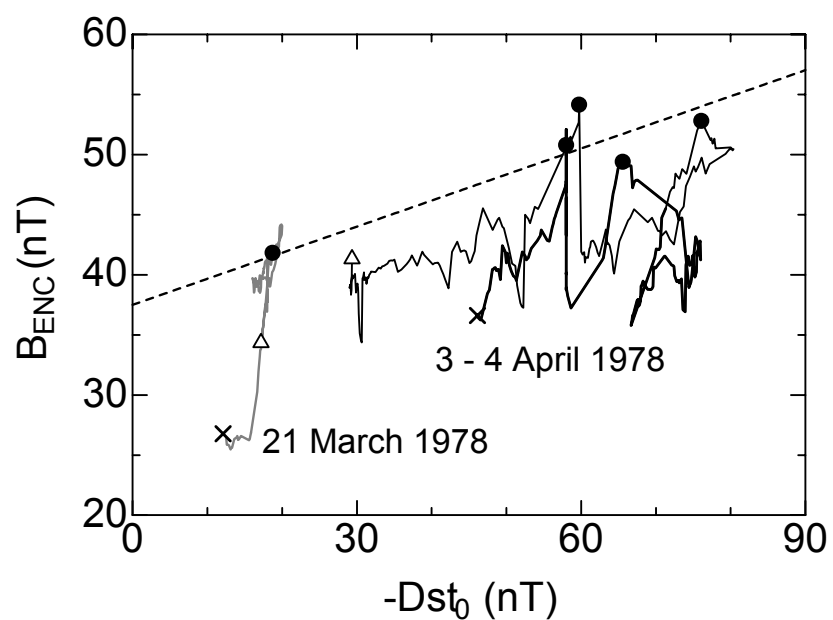

Fig. 10. Variations in $B_{E N C}$ values as a function of $D s t_{0}$ from 01:00 UT to 06:00 UT on 21 March 1978, and from 16:00 UT on 3 April to 08:00 UT on 4 April 1978. The thin and thick lines show the durations on 3 and 4 April, respectively. The gray line shows the duration on 21 March. The triangles and crosses indicate the start and end of the interval concerned, respectively. The solid circles represent the $B_{E N C}$ values just before the deflation events. The dashed line represents Eq. (7).

current during deflation events, the contributions from these two current systems are expected to compensate each other. Furthermore, on the basis of their statistical study, Nakai et al. $(1997,1999)$ argued that the partial ring current is enhanced in association with the occurrence of substorms. If this is the case, the contribution from the enhanced partial ring current can weaken the effect of the reducing tail current.

In conclusion, the $B_{E N C}$ value changes almost independently of the $D s t$ index during the deflation event, although the peak values of $B_{E N C}$ depend on the Dst index. In other words, the deflation event occurs preferably when the $B_{E N C}$ value reaches the critical value given by Eq. (7). Equation (7) suggests that when $D s t_{0}=-60 \mathrm{nT}$ the critical value is larger by $\sim 13 \mathrm{nT}$ than when $D s t_{0}=0 \mathrm{nT}$. Since the above model calculation estimates that the direct contribution from the ring current to the lobe field intensity is only $\sim 7 \mathrm{nT}$, the residual, i.e. an indirect influence of the ring current on the critical value, is probably not negligible. Thus, it seems likely that the cross-tail current can be more intensified with stronger ring currents before the onset of deflation events. This view appears to be rather reasonable, since the critical intensity of the cross-tail current must change considerably, depending on the outer and inner physical conditions. Nakai et al. (1999) suggested that the magnetic field magnitude near $X=\sim 20 R_{E}$ in the neutral sheet region increases with a decrease in Dst and/or an increase in the solar wind dynamic pressure. Since a small $B_{z}$ value in the neutral sheet region is one of the necessary conditions for triggering the tearing mode instability (e.g. Zelenyi et al., 1998), it is inferred that the formation of NENL is impeded when the ring current 
and/or the solar wind dynamic pressure is too strong. Under such conditions, the onset of the unloading process tends to be delayed, yielding that the lobe magnetic field becomes stronger before the unloading process begins.

\subsection{Northward turning of the IMF}

The northward turning of the IMF has been put forward as a possible external cause to trigger substorms (Lyons et al., 1997; Blanchard et al., 2000). Indeed, nine of the fourteen deflation events studied in the present study occurred when the IMF $B_{z}$ component was increasing. Let us examine again the 3-4 April events to inspect more closely the influences of the IMF. As we see in Fig. 5, the IMF $B_{z}$ component turned southward at 15:05 UT on 3 April and continued to be negative until 06:40 UT on 4 April. Four dipolarizations with deflations were observed during this period at 20:21 UT and 24:00 UT on 3 April and at 04:06 UT and 06:18 UT on 4 April. The first and third events occurred when the IMF $B_{z}$ component was decreasing or rather constant. It is difficult to evaluate conclusively whether the brief southward excursion in the IMF did indeed trigger the second event at 24:00 UT on 3 April. The fourth event seems to occur in association with an increase in the IMF $B_{z}$ component, although the variation in $B_{z}$ does not satisfy the "sharp northward turning" criterions defined by Lyons et al. (1997) and Blanchard et al. (2000). Therefore, a northward turning of the IMF or an increase in the IMF $B_{z}$ component is not necessarily a prerequisite for the occurrence of deflation events. It is not evident whether the northward turning was effective as a "preferable" condition for the 06:18 UT event. It is conceivable that the $B_{E N C}$ value was minimized at 04:27 UT, and increased to reach the critical value by $06: 18 \mathrm{UT}$, resulting in the occurrence of the next deflation event. Note, however, that the present observations do not deny the probability that the "sharp northward turning" or other external conditions can generate substorm expansions. Indeed, we have suggested that the 15 April event was triggered by a negative pulse in the IMF.

\subsection{Non-deflation events}

Although the deflation event is one of the most convincing evidence for the unloading process (Fairfield and Ness, 1970), substorm activity in terms of the $A L$ index is often enhanced without a deflation, as shown in Fig. 5. Some of them, such as the 09:53 UT event on 16 April, have not been identified as a deflation event simply because of a small decrease in the lobe field intensity, while the others, such as the 08:29 UT event on 21 March, did not show any reduction or sometimes showed increases in the lobe field intensity. Since the magnetic field pressure is approximately balanced in the azimuthal direction in the lobe, it is not conceivable that the magnetic field intensity decreased substantially in a localized region separated azimuthally from the spacecraft. Therefore, one of the possible interpretations for these events is that a deflation occurred far tailward of the spacecraft. In this case the lobe magnetic field is expected not to reduce, while the magnetic field dipolarization may propagate or expand earthward through the position of the spacecraft. Another possibility is that these dipolarizations and substorms actually occur without a deflation. This is not inconsistent with Taguchi et al. (1998), who showed that TCRs (traveling compression region) were observed only for 55 of 229 isolated substorm events, when the spacecraft was in the region $-38<X<-32 R_{E}$ in the midnight sector. Concentrating on the deflation events in the present study, we entrust further discussion on this issue to other papers. However, it is important to point out that the dipolarization in the near-Earth magnetotail is phenomenologically categorized into two groups: with a deflation and without a deflation, including the "gray" zone between these two categories, such as the 09:53 UT event of 16 April.

Acknowledgements. We greatly acknowledge C. T. Russell and O. W. Lennartsson for their kind permission for the use of ISEE-1 magnetic field and plasma data, respectively. We deeply thank the staff members at Institute of Geophysics and Planetary Physics on the University of California, Los Angeles (http://www-ssc. igpp.ucla.edu/forms/imp8_form.html) for the data from IMP-8, and World Data Center for Geomagnetism, Kyoto (http://swdcdb.kugi. kyoto-u.ac.jp/) for the $A L$ index. This work was carried out by the joint research program of the Solar-Terrestrial Environment Laboratory, Nagoya University.

Topical Editor G. Chanteur thanks Y. Maltser and another referee for their help in evaluating this paper.

\section{References}

Baker, D. N., Klimas, A. J., McPherron, R. L., and Büchner, J.: The evolution from weak to strong geomagnetic activity: An interpretation in terms of deterministic chaos, Geophys. Res. Lett., $17,41,1990$

Blanchard, G. T., Lyons, L. R., and Spann, J.: Prediction of substorms following northward turnings of the interplanetary magnetic field, J. Geophys. Res., 105, 375, 2000.

Burch, J. L.: Triggering of the substorm expansion phase by directional discontinuities in the interplanetary magnetic field, Geophys. Res. Lett., 6, 21, 1979.

Burton, R. K., McPherron, R. L., and Russell, C. T.: An empirical relationship between interplanetary conditions and Dst, J. Geophys. Res., 80, 4024, 1975.

Caan, M. N., McPherron, R. L., and Russell, C. T.: Substorm and interplanetary magnetic field effect on the geomagnetic tail lobes, J. Geophys. Res., 80, 191, 1975.

Fairfield, D. H. and Ness, N. F.: Configuration of the geomagnetic tail during substorms, J. Geophys. Res., 75, 7032, 1970.

Fairfield, D. H., Lepping, R. P., Hones, Jr., E. W., Bame, S., and Asbridge, J. R.: Simultaneous measurements of magnetotail dynamics by IMP spacecraft, J. Geophys. Res., 86, 1396, 1981.

Fairfield, D. H. and Jones, J.: Variability of the tail lobe field strength, J. Geophys. Res., 101, 7785, 1996.

Frank, L. A. and Craven, J. D.: Imaging result from Dynamics Explorer 1, Rev. Geophys., 26, 249, 1988.

Hones, Jr., E. W., Asbridge, J. R., Bame, S. J., and Singer, S.: Substorm variations of the magnetotail plasma sheet from $X_{S M} \approx$ $-6 R_{E}$ to $X_{S M} \approx-60 R_{E}$, J. Geophys. Res., 78, 109, 1973. 
Hones, Jr., E. W.: Transient phenomena in the magnetotail and their relation to substorms, Space Sci. Rev., 23, 393, 1979.

Ieda, A., Machida, S., Mukai, T., Saito, Y., Yamamoto, T., Nishida, A., Terasawa, T., and Kokubun, S.: Statistical analy of the plasmoid evolution with Geotail observations, J. Geophys. Res., 103, 4453, 1998.

Klimas, A. J., Baker, D. N., Roberts, D. A., Fairfield, D. H., and Büchner, J.: A nonlinear dynamical analogue model of geomagnetic activity, J. Geophys. Res., 97, 12 253, 1992.

Langel, R. A. and Estes, R. H.: Large-scale, near-field magnetic fields from external sources and the corresponding induced internal field, J. Geophys. Res., 90, 2487, 1985.

Lyons, L. R., Blanchard, G. T., Samson, J. C., Lepping, R. P., Yamamoto, T., and Moretto, T.: Coordinated observations demonstrating external substorm triggering, J. Geophys. Res., 102, 27 039, 1997.

Maezawa, K.: Magnetotail boundary motion associated with geomagnetic substorms, J. Geophys. Res., 80, 3543, 1975.

McPherron, R. L., Terasawa, T., and Nishida, A.: Solar wind triggering of substorm expansion onset, J. Geomag. Geoelectr., 38, 1089, 1986.

Nagai, T., Nakamura, R., Kokubun, S., Yamamoto, T., and Mukai, T.: Plasma sheet dynamics during substorms with Geotail observations, Substorms-4, (Eds) Kokubun, S. and Kamide, Y., 137, TERRAPUB, 1998.

Nakai, H., Kamide, Y., and Russell, C. T.: Influences of solar wind parameters and geomagnetic activity on the tail lobe magnetic field: A statistical study, J. Geophys. Res., 96, 5511, 1991.

Nakai, H. and Kamide, Y.: Estimating the long-term variations of the magnetotail pressure, J. Geophys. Res., 99, 23 747, 1994.

Nakai, H, Kamide, Y., and Russell, C. T.: Statistical nature of the magnetotail current in the near-Earth region, J. Geophys. Res.,
102, 9573, 1997.

Nakai, H, Kamide, Y., and Russell, C. T.: Dependence of the nearEarth magnetotail magnetic field on storm and substorm activities, J. Geophys. Res., 104, 22 701, 1999.

Ostapenko, A. A. and Maltsev, Y. P.: Storm time variation in the magnetospheric magnetic field, J. Geophys. Res., 105, 311, 2000.

Russell, C. T. and McPherron, R. L.: The magnetotail and substorms, Space Sci. Rev., 15, 205, 1973.

Russell, C. T.: The ISEE-1 and 2 fluxgate magnetometers, IEEE Trans. Geosci. Electron, GE-16, 239, 1978.

Shelley, E. G., Sharp, R. D., Johnson, R. G., Geiss, J., Eberhardt, P., Balsiger, H., Herendel, G., and Rosenbauer, H.: Plasma composition experiment on ISEE-A, IEEE Trans. Geosci. Electron., GE-16, 266, 1978.

Slavin, J. A., Smith, E. J., Tsurutani, B. T., et al.: Substorm associated traveling compression regions in the distant tail: ISEE-3 geotail observations, Geophys. Res. Lett., 11, 657, 1984.

Taguchi, S., Slavin, J. A., and R Lepping, P.: Traveling compression regions in the midtail: Fifteen years of IMP 8 observations, J. Geophys. Res., 103, 17 641, 1998.

Tsyganenko, N. A.: Magnetospheric magnetic field model with a warped tail current sheet, Planet. Space Sci., 37, 5, 1989.

Turner, N. E., Baker, D. N., Pulkkinen, T. I., and McPherron, R. L.: Evaluation of the tail current contribution to Dst, J. Geophys. Res., 105, 5431, 2000.

Valdivia, J. A., Sharma, A. S., and Papadopoulos, K.: Prediction of magnetic storms by nonlinear models, Geophys. Res. Lett., 23 , 2899, 1996.

Zelenyi, L. M., Petrukovichi, A. A., Budnick, E. Y., et al.: Substorm onset models and observations, Substorm-4, (Eds) Kokubun, S. and Kamide, Y., 327, TERRAPUB, 1998. 